\title{
Coughing May Lead to Spontaneous Chylothorax and Chylous Ascites
}

\author{
Gokhan Adas $^{\mathrm{a}}$ Oguzhan Karatepe ${ }^{\mathrm{a}} \quad$ Muharrem Battal $^{\mathrm{a}}$ \\ Yasar Dogan $^{\text {a }}$ Savas Karyagar ${ }^{\mathrm{b}}$ Asım Kutlu ${ }^{\mathrm{c}}$ \\ Departments of a General Surgery and ${ }^{b}$ Nuclear Medicine, Okmeydanı Training and \\ Research Hospital, and 'Department of Thoracic Surgery, Yedikule Training and \\ Research Hospital, Istanbul, Turkey
}

\section{Key Words}

Chylous ascites $\cdot$ Chylothorax $\cdot$ Lymph

\begin{abstract}
Chylous ascites is a rare form of ascites resulting from the accumulation of lymph in the abdominal cavity. It is due to an interruption in the lymphatic system. Surgical management is indicated in cases of recurrence or failure after conservative treatment. We report a case of spontaneous chylous peritonitis after chylothorax, which is a rare clinical event. The primary reason was unclear and the outcome of medical and surgical treatment was successful.
\end{abstract}

\section{Introduction}

Chylous ascites is the accumulation of a milk-like peritoneal fluid rich in triglycerides, due to the presence of thoracic, pericardium and intestinal lymph in the abdominal cavity. It is most commonly secondary to abdominal surgery, abdominal neoplasia, or trauma. Rarely, it can be a congenital event and spontaneous [1]. Management is based on identifying and treating the underlying cause. Very few cases of acute chylous peritonitis have been described in the literature. Since it is rarely seen, the primary diagnosis, treatment and especially the prognosis and the complications of chylous ascites are very important. We examined a patient in whom thoracic and peritoneal chylous ascites developed spontaneously and suddenly following a coughing seizure.

\section{Case Report}

A 33-year-old woman was admitted to the emergency department of a local hospital due to dyspenea and strong lateral pain on her right side which developed after a sudden coughing seizure. Right 
pleurisy was found on chest radiography and the patient was examined by an internal medicine specialist. Fluid was seen in the right pleural space by means of a thoracic USG and thoracentesis was performed. The thoracentesis fluid was chylous macroscopically and the level of triglycerides was 800 $\mathrm{mg} / \mathrm{dl}$ on biochemical analysis. The patient was diagnosed to have chylothorax and hospitalized at the clinic for thoracic disorders. No trauma, operation, or other disease was found in the anamnesis.

Right tube thoracostomy was performed since the primary etiology could not be determined by the Department of Thoracic Surgery. Total parenteral nutrition (TPN) was given as medical treatment for two weeks. Since the daily drainage was $500 \mathrm{ml}$ and the flow was not decreased as a result of the medical therapy, the patient was transferred to the Department of Thoracic Surgery after consultation. She was followed in this clinic for 10 days and oral nutrition was begun on the tenth day. Since the drainage was again $500 \mathrm{ml}$ as a result of oral nutrition, the decision to operate was made. The patient was operated and during the operation was incised in the thorax through the seventh intercostals by means of right lateral thoracotomy. When the right prevertebral area was explored after the pneumolysis, a leakage from the ductus thoracicus was detected at the top $5 \mathrm{~cm}$ away from the diaphragm. The main lymphatic channel (Ductus thoracicus) was sutured and ligatured and the drainage ceased. After the application of right posterior-inferior parietal pleurectomy, $5 \mathrm{ml}$ fibrin glue was applied to the region. Then the operation was terminated by placing a thoracal tube.

Since no fluid had come through the drain of the patient, who had begun oral nutrition on the first post-operative day, and also because the control radiographies of the chest were normal, the drain was removed. The patient, who had no post-operative problem, was discharged from the hospital on the fourth day of recovery. However, she complained of puffiness in the abdomen, distension and abdominal pain and was hospitalized again on the sixth post-operative day. On examination, her temperature was $38.5^{\circ} \mathrm{C}$, pulse rate was $90 / \mathrm{min}$, and blood pressure was $100 / 70 \mathrm{~mm} \mathrm{Hg}$. Systemic examination was unremarkable. The abdomen was soft and ovoid in shape, and there was diffuse sensitivity in all faces. Bowel sounds were audible. Blood analysis gave the following values: total leukocyte count $15.6 \times 10^{3} / \mu \mathrm{l}$; neutrophils $64.3 \%$; lymphocytes $22.7 \% \mathrm{Hb}, 143 \mathrm{~g} / \mathrm{l}$; platelet count $314000 / \mu \mathrm{l}$. Routine blood chemistry including liver function tests and white blood cell count were normal. Since chylous fluid was observed in the paracentesis and acute abdominal symptoms developed, a decision to operate was made and laparoscopic exploration was done. When entering the peritoneum, we noted a large amount of milky fluid in the peritoneal cavity. Chylous ascites was first considered because of the previous history. An abundant amount of chylous ascites was observed as a result of this exploration. No pathology was found at the intra-abdominal organs. The cell count, culture, gram's stain, total protein concentration, albumin, glucose, $\mathrm{LDH}$, amylase, triglyceride level, tuberculosis smear, culture, adenosine deaminase, and cytology were examined in the ascitic fluid. Chylous ascites of approximately 2,000 $\mathrm{ml}$ was drained, and the operation was terminated by inserting two drains (Winslow and Douglas) into the space of the abdomen. The patient was transferred to the Department of General Surgery. Oral nutrition was stopped and medical therapy was begun again. The daily drainage of $1,200 \mathrm{ml}$ decreased to $500 \mathrm{ml}$ on the tenth day. Since the general status and the biochemical parameters were found to be normal, and the drainage was less than $100 \mathrm{ml}$ per day, the patient was discharged from the hospital. She was requested to come for the post-operative weekly controls, and the daily lymphatic drainage was not less than $100 \mathrm{ml}$ per day in these controls.

Afterwards, the patient again had complaints such as puffiness and abdominal pain, and had daily flow at levels of 300-400 ml which again required hospitalization to the Department of General Surgery. Medical therapy (TPN + somatostatin analogue) was applied again for three weeks. Lymphoscintigraphy was requested. A lymphatic leakage from cysternae chyli was found at the level of L-2 in the scintigraphy that was taken again and a decision to operate was made (fig. 1, fig. 2 , fig. 3 ). The gamma camera was used during the operation in order to detect the location of the chylous fistule and due to the anatomical difficulties. Non-colloidal technetium $99 \mathrm{~m}$ was administered intravenously $30 \mathrm{~min}$ before the operation for this purpose. The cysterna chyli was found approximately at the level of L-2 at the right side of the aorta in the explorative laparotomy. $2 \mathrm{~cm}$ of the cysternae chyli was dissected and ligated and no leakage was seen during the $30 \mathrm{~min}$. By inserting drains to Winslow and Douglas regions, the operation was terminated. Postoperative drainage was $<100 \mathrm{ml}$ in the first week. The patient commenced a low-fat diet and was well recovered at 12-month follow-up. We have not seen any serious problem or complication in the patient that we have observed over a 36-month period. 


\section{Discussion}

Ascites is the presence of excess fluid in the peritoneal cavity. The concentration of ascites fluid was $5.48 \mathrm{mmol} / \mathrm{l}$, much higher than the blood triglyceride concentration of $102 \mathrm{mg} / \mathrm{dl}$. The basal flow rate of lymphatic fluid through the thoracic duct averages about $1.0 \mathrm{ml} / \mathrm{kg} / \mathrm{h}$, with a total volume of $1,500-1,700 \mathrm{ml} /$ day. Chylous ascites was defined as the presence of ascitic fluid with a fat (triglyceride) content higher than $200 \mathrm{mg} / \mathrm{dl}$ [1, 2]. Chylothorax is the most frequent cause of pleural effusion in fetuses and neonates. However, in adults it accounts for only $3 \%$ of cases of pleural effusion. The main causes of chylothorax are malignant tumors, $75 \%$ of which are lymphomas. Other rare causes are lymphangiomyomatosis, sarcoidosis, tuberculosis, venous thrombosis, congenital lymphatic malformations, trauma, nephrotic syndrome, hypothyroidism, cirrhosis, decompensated heart failure and idiopathic chylothorax. Chylothorax in adults is a rare clinical condition, and the diagnosis is made by ruling out other causes [1]. Although the etiology of spontaneous idiopathic chylothorax in adults is unknown, hyperextension of the neck is a likely suspect. The majority of spontaneous chylothorax cases are associated with minor traumas such as coughing (as the present case), vomiting, stretching or hiccups after a meal rich in fat $[3,5]$.

In the case described, a temporal relationship between cough and the appearance of lymphatic effusion, together with the absence of a specific cause, must be noted, which led to the diagnosis of spontaneous chylothorax. This case report does not only represent a very rare case of spontaneous chylous ascites, but also the rare coexistence of chylous ascites and chylothorax in an adult.

Chylous ascites frequently presents as progressive and painless abdominal distention, occurring over the course of weeks to months depending upon the underlying cause. Patients who have undergone abdominal or thoracic surgery may present with an acute onset [6-8]. The clinical presentation of an acute abdomen is less common. Free chyle is relatively non-irritating to the serosal surface, but pain may result from stretching of the retroperitoneum and mesenteric serosa, which may be responsible for the physical findings suggestive of an acute abdomen and subsequent laparoscopic exploration and drainage in our case.

The diagnosis of chylous ascites was confirmed by computed tomography. Its use is important in the detection of intraabdominal lymph nodes and masses. Especially in the cases of damage to the ductus thoracicus, it has great importance in determining the localization of the fluids. Other radiologic studies are lymphangiography and lymphangioscintigraphy. Specifically, these studies should be applied to patients for whom surgical procedures are thought necessary in order to determine leakage localization $[9,11]$.

The best treatment for chyloperitoneum is to correct the underlying cause if it can be identified. The patient should be examined carefully and should be prepared before the surgery. In many circumstances, the volume of the ascites can be controlled adequately using dietary restriction, parental nutrition, and paracentesis, but the management of persistent ascites is more difficult [10]. Preference for surgery should be in the cases where chylous ascites is resistant to medical therapy, and in cases that do not respond to temporary therapies such as lymphagiomatosis. In trauma and postoperative cases, surgery can be applied for positive results. The success rate for this is $75 \%$. However, complication rates are higher. 


\begin{tabular}{c|l|l|l}
$\begin{array}{r}\text { Case Reports in } \\
\text { Gastroenterology }\end{array}$ & $\begin{array}{l}\text { Case Rep Gastroenterol 2007;1:178-183 } \\
\text { D0I: 10.1159/000108950 }\end{array}$ & Published online: December 31, 2007 & $\begin{array}{l}\text { O 2007 S. Karger AG, Basel } \\
\text { ISSN 1662-0631 } \\
\text { www.karger.com/crg }\end{array}$ \\
\hline
\end{tabular}

Briefly, this case report aims to emphasize the presence of a rare presentation of a spontaneous coexistence of chylothorax and chylous ascites and the potential benefit of using a gamma camera and methylene blue dye to localize leakage.

Fig. 1. In the Tc-99m lymphoscintigraphy, for the explorations especially done for the ductus thoracicus, lymphatic drainage was observed in the upper part of the $10 \mathrm{~cm}$ of the umbilical region (around the celiac region).
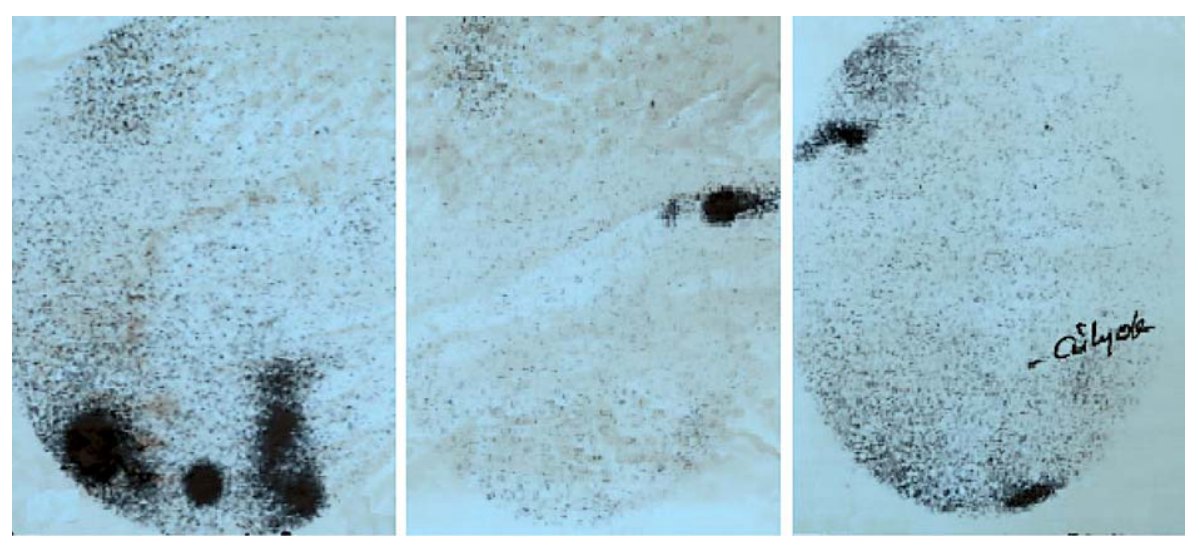

Fig. 2. A lymphatic leakage from cysternae chyli was found at the level of L-2 in the scintigraphy.

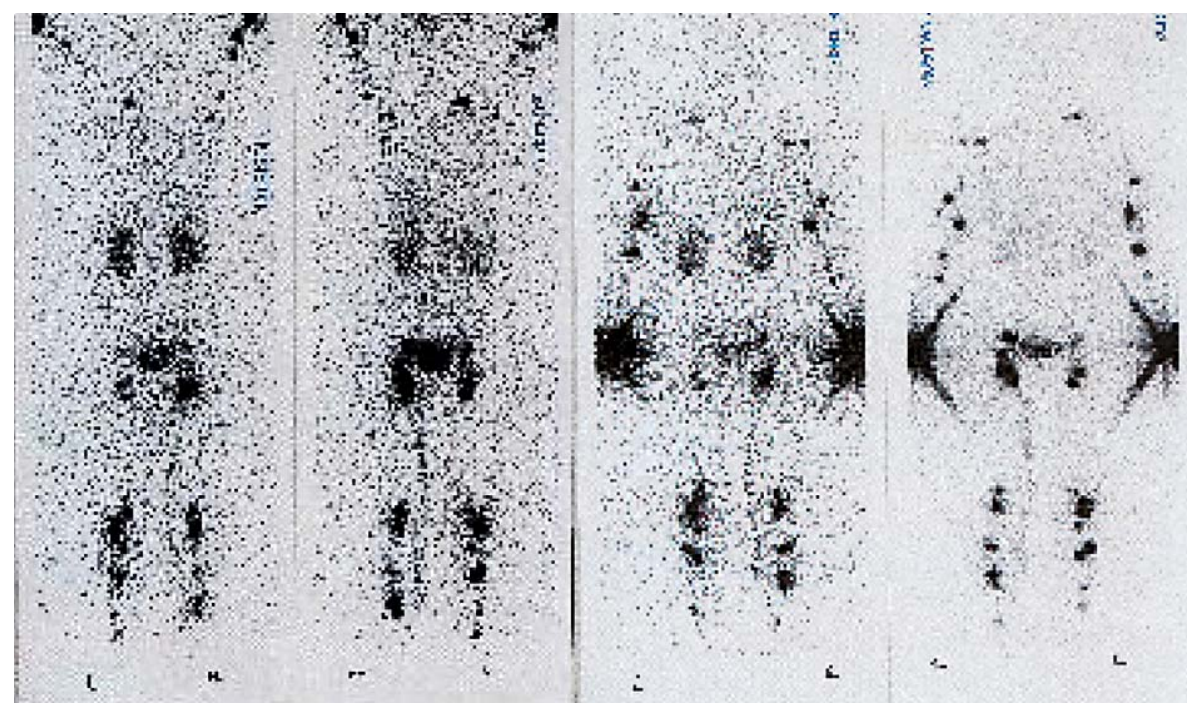


Fig. 3. Lymphatic stasis and compact popliteal and inguinal uptake of scintigraphic material. In the main lymphatic channel at the L1-L2 level (lymphatic obstruction at the level of cysternae), it was seen that lymphatic flow was ceased. An uptake was seen at the right axillar region due to collateral circulation.

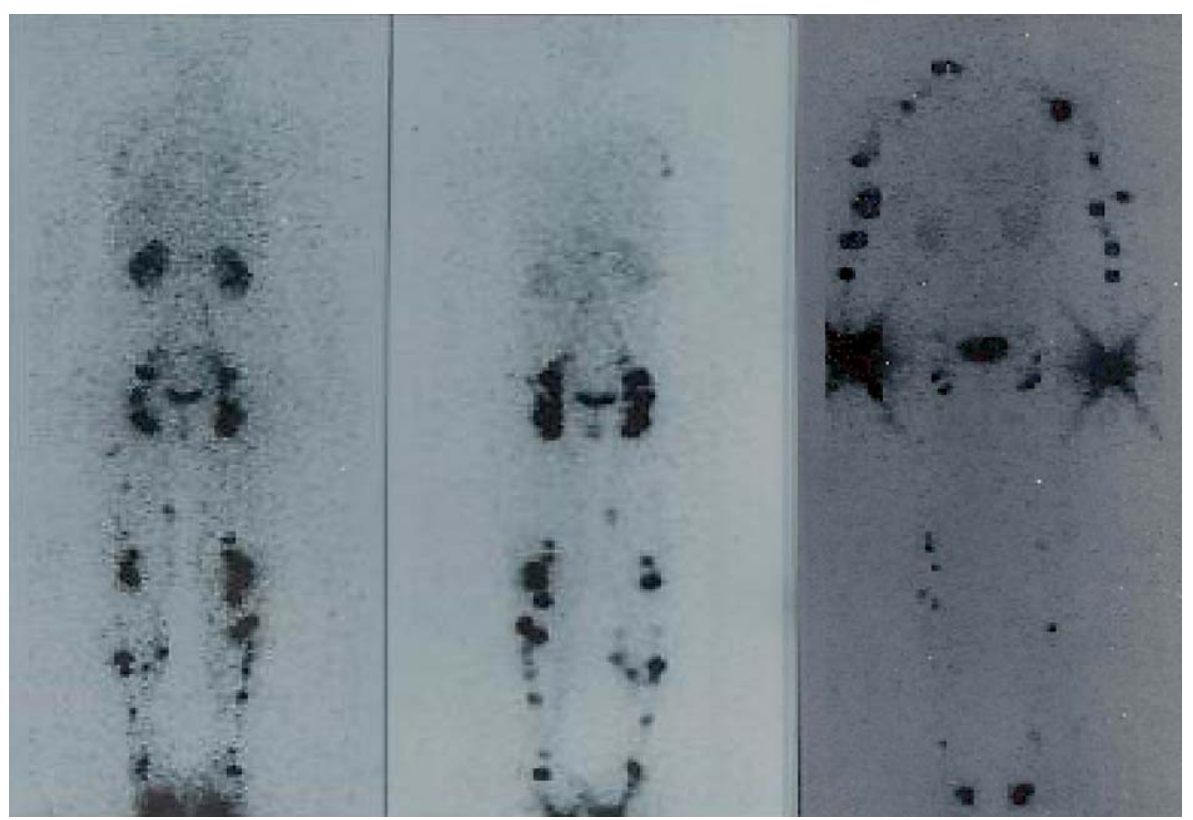




\section{References}

1 Madaniah AA: Spontaneous idiopathic chylothorax in adults. Saudi Med J 2005;26:145-146.

2 Aalami OO, Allen DB, Organ CH Jr: Chylous ascites: a collective review. Surgery 2000;128:761-778.

-3 Cardenas A, Chopra S: Chylous ascites. Am J Gastroenterol 2002;97:1896-1900.

-4 Almakdisı T, Massoud S, Makdisı G: Lymphomas and chylous ascites: review of the literature. Oncologist 2005;10:632-635.

-5 Huang Q, Jiang ZW, Jiang J, Li N, Li JS: Chylous ascites: treated with total parenteral nutrition and somatostatin. World J Gastroenterol 2004;10:2588-2591.

-6 Browse N, Wilson N, Rousso F, Al-Hassan H, Allen DR: Aetiology and treatment of chylous ascites. Br J Surg 1992;79:1145-1150.

7 Ridruejo E, Mando OG: Chylous ascites as the manifestation of left ventricular dysfunction: a case report. BMC Gastroenterol 2005;5:25.

-8 Fang, Hsu SD, Chen CW, Chen TW: Spontaneous chylous peritonitis mimicking acute appendicitis: a case report and review of literature. World J Gastroenterol 2006;12:154-156.

-9 Uriz J, Cardenas A, Arroyo V: Pathophysiology, diagnosis and treatment of ascites in cirrhosis. Baillieres Best Pract Res Clin Gastroenterol 2000;14:927-943.

10 Light RW: Chylothorax and pseudochylothorax; in Light RW (ed): Pleural Diseases, ed 4. Baltimore, Williams \& Wilkins, 1995, pp 284-298.

$\checkmark 11$ Pui MH, Yueh TC: Lymphoscintigraphy in chyluria, chyloperitoneum and chylothorax. J Nucl Med 1998;39:1292-1296. 